\title{
Sustentabilidade nos estabelecimentos assistenciais de saúde (EAS) e o COVID-19
}

\author{
Drielle Sanchez Leitner \\ Engenheira civil, doutoranda PPGEC UFPR \\ $\triangle$ drielle.dsl@gmail.com \\ Sergio Fernando Tavares \\ Professor doutor PPGEC UFPR \\ Adriana de Paula Lacerda Santos \\ Professora doutora PPGEC UFPR
}

\begin{abstract}
Resumo:
É crescente a busca pela sustentabilidade nas edificações. No caso de estabelecimento assistencial de saúde (EAS) esse esforço pode tornar-se ainda mais importante devido ao seu porte e complexidade. Apesar disso, no Brasil, não existem normativas especificas para a sustentabilidade na construção e operação de EAS. A fim de preencher essa lacuna a Agência Nacional de Vigilância Sanitária (ANVISA) dispõe em seu site publicações orientativas para a engenharia e arquitetura de EAS. Sabendo disso, o presente artigo visou identificar, dentro dos documentos ranqueados pela ANVISA, informações que pudessem ser relacionadas a busca pela sustentabilidade dos EAS, englobando suas esferas econômica, social e ambiental. Como resultado, os documentos apresentaram individualmente informações sobre pelo menos uma das esferas, sendo que quatro deles apresentaram informações sobre as três esferas. Adicionalmente, pretendeu-se localizar, considerando os mesmos documentos, fatores inerentes aos EAS que colaboram para o combate do COVID-19, pandemia mundial que chama mais atenção para os EAS e seu possível potencial no auxílio da resolução dessa situação. Como resultado, foram destacadas ações de higiene, limpeza, imunização, compartimentação da edificação e barreiras para transmissão. Por fim, foi discutida sobre a importância de ações que preencham os requisitos das três esferas da sustentabilidade simultaneamente e como isso pode resultar em uma edificação mais eficiente na resolução de situações imprevistas.

Palavras-chave: Estabelecimento assistencial de saúde (EAS), ANVISA, sustentabilidade, pilares, COVID-19.
\end{abstract}

\section{Sustainability in healthcare facilities and COVID-19}

\begin{abstract}
:
The search for sustainability in buildings is on the grown. In the case of healthcare facilities (EAS), this effort can become even more important due to its size and complexity. Despite this, in Brazil, there are no specific regulations for sustainability in the construction and operation of EAS. In order to fill this gap, the National Health Surveillance Agency (ANVISA) has on its website publications that guide the engineering and architecture of EAS. Knowing this, this article aimed to identify, within the documents ranked by ANVISA, information that could be related to the search for sustainability in EAS, encompassing their economic, social, and environmental spheres. As a result, the documents
\end{abstract}


individually presented information on at least one of the spheres, with four of them presenting information on the three spheres. Additionally, it was intended to locate, considering the same documents, factors inherent to EAS that collaborate to combat COVID-19, a global pandemic that draws more attention to EAS and its possible potential to help resolve this situation. As a result, hygiene, cleaning, immunization, building partitioning and transmission barriers were highlighted. Finally, it was discussed the importance of actions that meet the requirements of the three spheres of sustainability simultaneously and how this can result in a more efficient building in solving unforeseen situations.

Keywords: Healthcare facilities, ANVISA, sustainability, pillars, COVID-19.

\section{La sostenibilidad en los establecimientos de salud y COVID-19}

\section{Resumen:}

Crece la búsqueda de la sostenibilidad en los edificios. En el caso de los establecimientos de salud (EAS), este esfuerzo puede llegar a ser aún más importante por su tamaño y complejidad. A pesar de esto, en Brasil, no existen regulaciones específicas para la sostenibilidad en la construcción y operación de EAS. Para llenar este vacío, la Agencia Nacional de Vigilancia Sanitaria (ANVISA) tiene en su sitio web publicaciones que orientan la ingeniería y arquitectura de EAS. Sabiendo esto, este artículo tuvo como objetivo identificar, dentro de los documentos clasificados por ANVISA, información que pudiera estar relacionada con la búsqueda de la sostenibilidad de los EAS, abarcando sus ámbitos económico, social y ambiental. Como resultado, los documentos presentaron información individualmente sobre al menos una de las esferas, y cuatro de ellos presentaron información sobre las tres esferas. Adicionalmente, se pretendía ubicar, considerando los mismos documentos, factores inherentes a EAS que colaboran para combatir COVID-19, una pandemia global que llama más la atención sobre los EAS y su posible potencial para ayudar a resolver esta situación. Como resultado, se destacaron la higiene, la limpieza, la inmunización, la división de edificios y las barreras de transmisión. Finalmente, se discutió la importancia de acciones que cumplan con los requerimientos de los tres ámbitos de la sustentabilidad simultáneamente y cómo esto puede resultar en un edificio más eficiente en la resolución de situaciones imprevistas.

Palabras clave: Establecimiento de salud, ANVISA, sostenibilidad, pilares, COVID-19.

\section{INTRODUÇÃO}

O termo sustentabilidade refere-se ao conjunto de processos destinados a manter a vitalidade e integridade da Terra e assim a preservação dos seus ecossistemas com seus respectivos elementos físicos, químicos e ecológicos. Busca-se dessa forma possibilitar a existência da vida, o atendimento das necessidades presentes e futuras, e a continuidade e expansão da civilização humana (ONU, 2000).

Para que a sustentabilidade real seja alcançada ela deve estar presente em todas as práticas humanas (ONU, 2000). Nesse contexto, Elkington (1994) propôs a divisão do conceito da sustentabilidade em três esferas, ou pilares, sendo elas: ambiental, econômica e social. Assim, para atingir a sustentabilidade deve-se alcançar o equilíbrio entre seus três pilares, que podem ser definidos como (SACHS, 1993): 
- Sustentabilidade social: é a capacidade de proporcionar qualidade de vida, criando comunidades saudáveis e habitáveis com base em equidade, diversidade, conectividade e democracia;

- Sustentabilidade econômica: representa o ideal emprego dos recursos existentes, para que seja possível alcançar um equilíbrio responsável e a preservação do capital;

- Sustentabilidade ambiental: é a capacidade de usar recursos naturais sem exceder sua capacidade regenerativa e proteger o "capital natural" para evitar danos aos seres humanos e o meio ambiente.

A construção civil não foge do tema sustentabilidade, e sim o oposto. Segundo a Agenda 21 do International Council for Research and Innovation in Building and Construction (CIB), a indústria da construção e o ambiente construído são fundamentais para o desenvolvimento sustentável da sociedade (CIB, 1999). Nesse contexto surgiram diversos documentos buscando um pensamento mais sustentável dentro da cadeia da construção civil, como publicação da Agenda 21, e ferramentas de avaliação das edificações como o Building Research Establishment Environmental Assessment Method (BREEAM), criado no Reino Unido em 1990 (VILHENA, 2007). As próprias normas, quando alinhadas com o conceito de sustentabilidade e desenvolvimento sustentável, podem apresentar itens que almejem uma construção, e posteriormente uma edificação, mais sustentável. Como exemplo, a norma NBR 15575-1, que trata do desempenho de edificações habitacionais, cita a sustentabilidade como exigência do usuário englobando a durabilidade, a manutenibilidade e os impactos ambientais da edificação (ABNT, 2013).

No caso das edificações assistenciais de saúde (EAS), a questão da sustentabilidade fica distante visto a falta de políticas públicas relativas à gestão e planejamento dos EAS, como relatado no contexto das unidades de urgência e emergência por Cavalcanti et al. (2019). A norma em vigor, mas em revisão (CAVALCANTI et al., 2019), é a Resolução da Diretoria Colegiada (RDC) № 50/2002 que dispõe sobre o regulamento técnico para planejamento, programação, elaboração e avaliação de projetos físicos de EAS (BRASIL, 2002). 0 documento em questão não contempla a questão da sustentabilidade em EAS.

Visando preencher a lacuna de publicações especificas para construções de EAS, a Agência Nacional de Vigilância Sanitária (ANVISA) dispõe em seu site uma lista com dez publicações que podem ser utilizadas na construção e operação de EAS. Os documentos são 
orientativos sendo que os critérios podem ser conflitantes entre si, cabendo ao responsável avaliar aqueles mais adequados a sua situação.

Ainda nesse contexto, ressalta-se a importância dos EAS no cuidado e manutenção da vida podendo ser associado a sustentabilidade social e ao Objetivo de Desenvolvimento Sustentável (ODS) 3, referente a saúde e ao bem-estar, proposto pela Agenda 2030 da ONU (ONU, 2015). Soma-se ao cenário a questão da pandemia mundial de saúde, o COVID-19, com alto número de contaminados e mortos, crise no sistema de saúde nacional e internacional, sendo que no âmbito da construção civil também têm sido discutidos os cuidados necessários para evitar a transmissão (BONATTO, 2020), além do possível potencial das EAS em auxiliar, mesmo que parcialmente, na contenção da pandemia.

Considerando as questões apresentadas, o presente artigo tem como objetivo identificar, dentro dos documentos ranqueados pela ANVISA, informações que possam ser relacionadas a busca pela sustentabilidade dos EAS englobando suas três esferas. Adicionalmente, pretende-se ressaltar, tendo em mente os mesmos documentos, os fatores inerentes as construções hospitalares e suas características arquitetônicas os quais colaboram para o combate da pandemia do COVID-19. Assim, visa-se responder a seguinte pergunta de pesquisa: como os manuais da ANVISA referentes a engenharia e arquitetura de EAS englobam em seus escopos as questões da sustentabilidade econômica, social e ambiental? Além disso, considerando o COVID-19, visa-se identificar se os manuais possuem indicações que auxiliem na resolução dessa situação, mesmo que parcialmente.

\section{METODOLOGIA}

O presente estudo iniciou-se pela identificação de documentos a serem seguidos na elaboração de EAS, buscando, principalmente, informações quanto a sustentabilidade desses. Seguindo essa etapa, foi localizado no site da ANVISA manuais para engenharia e arquitetura de edificações desse tipo (ANVISA, 2020), contribuindo para o estabelecimento do objetivo. Visou-se então proporcionar maior familiaridade com o tema, sendo seu objetivo exploratório (GIL, 2010). A estratégia da pesquisa é a pesquisa bibliográfica, englobando principalmente os documentos indicados pela ANVISA, mas também artigos atuais, 
configurando o estado da arte do assunto em questão. Ademais, a pesquisa possui abordagem majoritariamente qualitativa, buscando informações para o desenvolvimento de um panorama sobre $o$ assunto.

As etapas seguidas se assemelham a de Bernardi et al. (2017) que primeiramente descreveram os documentos em estudo e em um segundo momento relacionaram as informações encontradas. Ademais, a associação entre o estudo das esferas da sustentabilidade e as edificações hospitalares foi realizada anteriormente por Froehlich e Bitencourt (2015) em um estudo de caso sobre o modelo de gestão do Hospital Mãe de Deus (Porto Alegre).

De forma a deixar os resultados da pesquisa mais claros e organizados, para o desenvolvimento da extração das informações dos documentos foi seguida a lógica da ferramenta 5W1H (Who-What-When-Where-Why-How), ferramenta da qualidade que permite a rápida identificação dos elementos intrínsecos de um projeto, aplicada em publicações recentes, como Lee et al. (2019), Leitner, Sotsek e Santos (2020) e Tang et al. (2019b). Seguindo a ferramenta foram feitas perguntas a fim de organizar a extração das informações e então os resultados, como mostra a Tabela 1.

Tabela 1 - Aplicação da ferramenta 5W1H

\begin{tabular}{ccc}
\hline Categorias 5W1H & Perguntas 5W1H & Respostas 5W1H \\
Where & Qual o foco de aplicação da publicação? & EAS \\
Who & Qual a publicação que está sendo estudada? & Documento \\
When & Qual a data da publicação? & Data \\
Why & Qual o objetivo da publicação? & Objetivo / escopo \\
What & Qual pilar da sustentabilidade (ou CoVID-19) foi englobado? & Pilar / CoVID-19 \\
How & Como o assunto foi englobado? & Ações identificadas \\
\hline
\end{tabular}

Fonte: Os autores, 2021.

\section{RESULTADOS E DISCUSSÃo}

Seguindo a lógica da ferramenta $5 \mathrm{~W} 1 \mathrm{H}$, a partir da leitura e entendimento dos documentos do portal da ANVISA quanto a arquitetura e engenharia de EAS, foram 
elaboradas as Tabelas 2 e 3. A Tabela 2 apresenta os documentos que fizeram parte da pesquisa, delimitando o campo "Who", as datas das publicações, delimitando o campo "When", além da descrição resumida dos documentos, possibilitando posteriormente fazer a extração das informações pertinentes de forma a cumprir os objetivos da pesquisa, caracterizando assim a questão "Why”.

Tabela 2 - Manuais de arquitetura e engenharia hospitalar - ANVISA

\begin{tabular}{|c|c|c|}
\hline WHO - Documento & $\begin{array}{l}\text { WHEN - } \\
\text { Fonte }\end{array}$ & WHY - Objetivo \\
\hline $\begin{array}{c}\text { Arquitetura na Prevenção } \\
\text { de Infecção Hospitalar }\end{array}$ & $\begin{array}{c}\text { Brasil } \\
(1995 a)\end{array}$ & Abrange técnicas de controle de infecção dentro de EAS. \\
\hline $\begin{array}{l}\text { Condições Ambientais de } \\
\text { Leitura Visual }\end{array}$ & $\begin{array}{c}\text { Brasil } \\
(1995 b)\end{array}$ & $\begin{array}{c}\text { Discorre sobre o suporte cognitivo e conforto visual do edifício } \\
\text { englobando a orientabilidade e a identificabilidade de } \\
\text { informações e as relações da edificação com seu entorno. }\end{array}$ \\
\hline $\begin{array}{l}\text { Condições de Segurança } \\
\text { Contra Incêndio }\end{array}$ & $\begin{array}{c}\text { Brasil } \\
(1995 c) \\
\end{array}$ & $\begin{array}{c}\text { Tem foco na prevenção de incêndios com a proteção da vida } \\
\text { humana e dos bens patrimoniais. }\end{array}$ \\
\hline $\begin{array}{l}\text { Instalações Prediais } \\
\text { Ordinárias e Especiais }\end{array}$ & $\begin{array}{l}\text { Brasil } \\
(1995 d)\end{array}$ & $\begin{array}{c}\text { Orienta e fixa condições técnicas para elaboração de projetos } \\
\text { elétricos, eletrônicos, hidráulicos, fluidos mecânicos e } \\
\text { climatização de EAS. }\end{array}$ \\
\hline $\begin{array}{c}\text { Manutenção Incorporada à } \\
\text { Arquitetura Hospitalar }\end{array}$ & $\begin{array}{c}\text { Brasil } \\
(1995 e) \\
\end{array}$ & $\begin{array}{l}\text { Discorre sobre a manutenção de um EAS com a criação de } \\
\text { espaços destinados exclusivamente à manutenção. }\end{array}$ \\
\hline $\begin{array}{c}\text { O Custo das Decisões } \\
\text { Arquitetônicas no Projeto } \\
\text { de Hospitais }\end{array}$ & $\begin{array}{c}\text { Brasil } \\
(1995 f)\end{array}$ & $\begin{array}{l}\text { Apresenta como as decisões tomadas num projeto de } \\
\text { arquitetura de um EAS tem relação com o custo final da obra. }\end{array}$ \\
\hline $\begin{array}{l}\text { Sistemas de Controle das } \\
\text { Condições Ambientais de } \\
\text { Conforto }\end{array}$ & $\begin{array}{c}\text { Brasil } \\
(1995 g)\end{array}$ & $\begin{array}{c}\text { Discorre sobre a questão do conforto luminoso, acústico e } \\
\text { térmico no interior de um EAS. }\end{array}$ \\
\hline $\begin{array}{l}\text { Sistemas Construtivos na } \\
\text { Programação Arquitetônica } \\
\text { de Edifícios de Saúde }\end{array}$ & $\begin{array}{c}\text { Brasil } \\
(1995 \mathrm{~h})\end{array}$ & $\begin{array}{l}\text { Identifica critérios que possam contribuir para a melhoria de } \\
\text { qualidade das decisões com relação ao sistema construtivo a ser } \\
\text { adotado no projeto de EAS. }\end{array}$ \\
\hline $\begin{array}{l}\text { Segurança no Ambiente } \\
\text { Hospitalar }\end{array}$ & $\begin{array}{l}\text { Brasil } \\
(2003) \\
\end{array}$ & Apresenta informações sobre os principais riscos nos EAS. \\
\hline RDC Anvisa № 306/ 2004 & $\begin{array}{l}\text { Brasil } \\
(2004)\end{array}$ & Dispõe sobre o gerenciamento de resíduos em EAS. \\
\hline
\end{tabular}

Fonte: Os autores, 2021.

Como pode ser observada, a distribuição temporal das publicações, questão "When", encontra-se em um período menor do que uma década (de 1995 a 2004). Além disso, as publicações chegam a ultrapassar duas décadas de lançamento, podendo estar defasadas e indicando uma lacuna de atualização do assunto. Quanto aos objetivos das publicações, questão "Why", esses foram variados englobando desde o conforto do ocupante até questões técnicas de projeto. O assunto "Where" não foi incluído na tabela uma vez que todos os documentos estão relacionados com EAS, sendo essa a resposta global da questão, seguindo o objetivo do presente artigo. 
Quanto a identificação dos pilares da sustentabilidade e de informações úteis para ao combate do COVID-19, questões "What" e "How', a Tabela 3 mostra o resumo dos resultados encontrados por publicação. Como pode ser observado, dos dez documentos, quatro apresentaram características pela busca das três esferas da sustentabilidade. Dessa forma, esses quatro documentos demonstraram preocupação em alcançar a sustentabilidade real da edificação (BRASIL, 1995c, 1995d, 1995g, 2003).

Tabela 3 - Características "What" e "How" identificadas nos documentos

\begin{tabular}{|c|c|c|c|c|c|}
\hline \multirow{3}{*}{$\begin{array}{l}\text { WHO/ WHEN - } \\
\text { Documento }\end{array}$} & & \multicolumn{4}{|c|}{ WHAT } \\
\hline & & \multicolumn{3}{|c|}{ Sustentabilidade } & \multirow{2}{*}{ COVID-19 } \\
\hline & & Ambiental & Econômica & Social & \\
\hline $\begin{array}{l}\text { Arquitetura na } \\
\text { prevenção de } \\
\text { infecção } \\
\text { hospitalar } \\
\text { (BRASIL, 1995a) }\end{array}$ & \multirow{8}{*}{ 茥 } & - & $\begin{array}{c}\text { Vantagens } \\
\text { econômicas da } \\
\text { instalação de um } \\
\text { tubulão }\end{array}$ & $\begin{array}{l}\text { Cuidado e } \\
\text { prevenção de } \\
\text { infecções } \\
\text { hospitalares }\end{array}$ & $\begin{array}{c}\text { Lavagem das mãos, } \\
\text { barreiras para a } \\
\text { transmissão }\end{array}$ \\
\hline $\begin{array}{c}\text { Condiçõoes } \\
\text { Ambientais de } \\
\text { Leitura Visual } \\
\text { (BRASIL, 1995b) }\end{array}$ & & $\begin{array}{c}\text { Acesso } \\
\text { diferenciado } \\
\text { para insumos } \\
\text { poluentes }\end{array}$ & - & $\begin{array}{c}\text { Suporte cognitivo } \\
\text { da edificação e } \\
\text { conexão com seu } \\
\text { entorno }\end{array}$ & $\begin{array}{c}\text { Setorização/ } \\
\text { compartimentação da } \\
\text { edificação }\end{array}$ \\
\hline $\begin{array}{c}\text { Condições de } \\
\text { Segurança Contra } \\
\text { Incêndio (BRASIL, } \\
\text { 1995c) }\end{array}$ & & $\begin{array}{l}\text { Evitar liberação } \\
\text { de gases } \\
\text { poluentes para } \\
\text { a atmosfera, } \\
\text { reconstruções e } \\
\text { propagação do } \\
\text { fogo para áreas } \\
\text { florestais }\end{array}$ & $\begin{array}{l}\text { Proteção de bens } \\
\text { (patrimônio) }\end{array}$ & $\begin{array}{c}\text { Proteção da vida } \\
\text { humana }\end{array}$ & $\begin{array}{c}\text { Barreiras para a } \\
\text { transmissão, escolha dos } \\
\text { revestimentos, } \\
\text { setorização/ } \\
\text { compartimentação da } \\
\text { edificação }\end{array}$ \\
\hline $\begin{array}{c}\text { Instalações } \\
\text { Prediais } \\
\text { Ordinárias e } \\
\text { Especiais (BRASIL, } \\
\text { 1995d) }\end{array}$ & & $\begin{array}{c}\text { Conservação de } \\
\text { energia }\end{array}$ & $\begin{array}{l}\text { Estudo do sistema } \\
\text { energético }\end{array}$ & $\begin{array}{l}\text { Promoção da } \\
\text { saúde }\end{array}$ & $\begin{array}{l}\text { Setorização do ar- } \\
\text { condicionado }\end{array}$ \\
\hline $\begin{array}{c}\text { Manutenção } \\
\text { Incorporada à } \\
\text { Arquitetura } \\
\text { Hospitalar } \\
\text { (BRASIL, 1995e) }\end{array}$ & & $\begin{array}{l}\text { Planejamento } \\
\text { preventivo de } \\
\text { riscos }\end{array}$ & $\begin{array}{c}\text { Planejamento } \\
\text { preventivo dos } \\
\text { custos e } \\
\text { dimensionament } \\
\text { o da rede elétrica }\end{array}$ & - & - \\
\hline $\begin{array}{c}\text { O Custo das } \\
\text { Decisões } \\
\text { Arquitetônicas no } \\
\text { Projeto de } \\
\text { Hospitais (BRASIL, } \\
\text { 1995f) }\end{array}$ & & - & $\begin{array}{l}\text { Influência da } \\
\text { arquitetura nos } \\
\text { custos }\end{array}$ & - & $\begin{array}{c}\text { Setorização/ } \\
\text { compartimentação da } \\
\text { edificação }\end{array}$ \\
\hline $\begin{array}{l}\text { Sistemas de } \\
\text { Controle das } \\
\text { Condições } \\
\text { Ambientais de } \\
\text { Conforto (BRASIL, } \\
\text { 1995g) }\end{array}$ & & $\begin{array}{l}\text { Amenização dos } \\
\text { impactos } \\
\text { causados ao } \\
\text { meio ambiente }\end{array}$ & $\begin{array}{c}\text { Custos de } \\
\text { materiais } \\
\text { isolantes } \\
\text { acústicos e custo } \\
\text { energético }\end{array}$ & $\begin{array}{l}\text { Conforto do } \\
\text { ocupante }\end{array}$ & - \\
\hline $\begin{array}{c}\text { Sistemas } \\
\text { Construtivos na }\end{array}$ & & - & $\begin{array}{c}\text { Uso de } \\
\text { construções }\end{array}$ & $\begin{array}{l}\text { Conforto do } \\
\text { ocupante }\end{array}$ & Escolha dos revestimentos \\
\hline
\end{tabular}




\begin{tabular}{|c|c|c|c|c|}
\hline $\begin{array}{c}\text { Programação } \\
\text { Arquitetônica de } \\
\text { Edifícios de Saúde } \\
\text { (BRASIL, 1995h) }\end{array}$ & & $\begin{array}{l}\text { modulares; } \\
\text { redução de custos } \\
\text { de manutenção e } \\
\text { de custos globais }\end{array}$ & & \\
\hline $\begin{array}{l}\text { Segurança no } \\
\text { Ambiente } \\
\text { Hospitalar } \\
\text { (BRASIL, 2003) }\end{array}$ & $\begin{array}{l}\text { Controle das } \\
\text { condições } \\
\text { radiológicas } \\
\text { evitando } \\
\text { contaminação } \\
\text { atmosférica }\end{array}$ & $\begin{array}{l}\text { Custo de: } \\
\text { acidentes, } \\
\text { esterilização, } \\
\text { manutenção, } \\
\text { equipamentos e } \\
\text { treinamento }\end{array}$ & $\begin{array}{c}\text { Segurança no } \\
\text { trabalho- } \\
\text { conservação da } \\
\text { vida do } \\
\text { funcionário e } \\
\text { demais ocupantes } \\
\end{array}$ & $\begin{array}{c}\text { Barreiras para a } \\
\text { transmissão, cuidados de } \\
\text { esterilização e desinfecção } \\
\text { hospitalar, setorização do } \\
\text { ar-condicionado, escolha } \\
\text { dos revestimentos }\end{array}$ \\
\hline $\begin{array}{l}\text { RDC ANVISA No } \\
\text { 306/2004 (BRASIL, } \\
\text { 2004) }\end{array}$ & $\begin{array}{c}\text { Preservar o } \\
\text { meio ambiente }\end{array}$ & - & $\begin{array}{l}\text { Preservar a saúde } \\
\text { pública }\end{array}$ & $\begin{array}{l}\text { Escolha dos } \\
\text { revestimentos, higiene, } \\
\text { equipamentos de proteção } \\
\text { individual, campanhas de } \\
\text { imunização }\end{array}$ \\
\hline
\end{tabular}

Fonte: Os autores, 2021.

Ainda sobre a busca pela sustentabilidade ambiental em EAS, verificou-se principalmente a atenção em evitar contaminações ambientais, sejam essas causados por resíduos (BRASIL, 1995b, 2004), gases utilizados nas atividades médicas (BRASIL, 2003), ou até mesmo incêndios, situação na qual o uso da abordagem correta poderia evitar a liberação de gases poluentes para a atmosfera, reconstruções e propagação do fogo para áreas florestais (BRASIL, 1995c). As informações localizadas estão de acordo com a meta 3.9 do ODS 3 de reduzir substancialmente, até 2030, o número de mortes e doenças por produtos químicos perigosos, contaminação e poluição do ar e água do solo (ONU, 2015).

O documento que se destacou pela sua preocupação com a sustentabilidade ambiental foi Brasil (2004), que visa minimizar a produção de resíduos além de proporcionar aos resíduos gerados o encaminhamento seguro, visando à proteção dos trabalhadores, a preservação da saúde pública e do meio ambiente. O destaque do documento pode estar relacionado com a sua data de publicação, uma vez que o documento seja o mais recente da lista.

Quanto a contextualização dessa esfera, o acesso diferenciado para insumos poluentes, discutido em Brasil (1995b), aparece em outras publicações como no Achieving Excellence Design Evaluation Toolkit (AEDET), documento desenvolvido no Reino Unido que propõem um sistema de avaliação integral de edifícios de saúde (GUELLI, 2006). Além disso, a questão dos resíduos, foco de Brasil (2004), está presente em diversos artigos atuais, entre os itens do tópico destacam-se: a separação, o armazenamento e o gerenciamento de resíduos (BRAMBILLA; CAPOLONGO, 2019; DE FÁTIMA CASTRO; MATEUS; BRAGRANÇA, 2017; RYAN-FOGARTY; 
O'REGAN; MOLES, 2016); e, a importância do desenvolvimento de um plano de gestão ambiental (BRAMBILLA; CAPOLONGO, 2019; DE FÁTIMA CASTRO; MATEUS; BRAGRANÇA, 2017). Ademais, a questão da poluição por radiação, tratada em Brasil (2003), é um dos itens presente no modelo de avaliação de desempenho de edificações hospitalares elaborado por Lobo (2010).

Traços pela busca pela sustentabilidade econômica foram localizados em oito das dez publicações, sendo que essas informações estavam mais bem definidas do que as da esfera anterior. Custos relativos à energia foram considerados em três casos (BRASIL, 1995d, 1995e, 1995g), sendo que a busca pela redução no consumo de energia, além de teor econômico pode também trazer benefícios ambientais. Nesse âmbito, Brasil (1995d) sugere o uso de materiais de construção inteligentes que possibilitem o controle e a redução do uso de energia.

O tema em questão também está presente em artigos atuais (PRINCIPI et al., 2016; RYAN-FOGARTY; O'REGAN; MOLES, 2016), como Principi et al. (2016) que avalia, por meio de simulações de desempenho, o consumo energético de cinco hospitais, apresentando estratégias de economia de energia como, por exemplo, o uso de paredes com isolamento térmico externo e a substituição de janelas, o que está de acordo com a sugestão de Brasil (1995d). Ainda, no caso de Brasil (1995e), é citada a questão do correto dimensionamento da rede elétrica visando reduzir distancias e assim reduzir potenciais perdas.

Vantagens econômicas relacionadas a questões construtivas também fizeram parte das publicações (BRASIL, 1995a, 1995f, 1995g, 1995h). Brasil (1995a) cita os possíveis benefícios econômicos da instalação de um tubulão para a retirada de roupas sujas, ação que também contribuiria para a esfera social uma vez que exporia menos os funcionários a riscos envolvidos nessa atividade. Em, Brasil (1995g) a sustentabilidade econômica é discutida no aspecto dos custos de materiais isolantes acústicos. Já no caso de Brasil (1995f), que tem enfoque exclusivamente econômico, é debatido sobre a influência da volumetria, considerando características como altura e função do cômodo, nos custos da construção da EAS. Por fim, Brasil (1995h) foca na escolha do sistema construtivo do EAS, englobando: definições do projeto básico com a estimativa de consumo de materiais, a quantificação e custos de serviços e fornecimentos; vantagens do uso de construções modulares no tempo e custos de construção; redução de custos globais de obra, sem prejuízo das exigências gerais de qualidade construtiva; e, redução de custos da manutenção. 
Os custos de manutenção, incluídos em Brasil (1995h), também fizeram parte de Brasil (2003) e de pesquisas mais recentes sobre o tópico (REIJULA; REIJULA; REIJULA, 2016). Outros itens como os custos de treinamento e reciclagem para os funcionários e despesas envolvidas na desinfecção de áreas e equipamentos, também estão presentes em Brasil (2003). Sobre o uso de sistemas construtivos modulares e pré-fabricados, levantado por Brasil (1995h), foram localizadas outras publicações que discorrem sobre as vantagens desses em EAS, abrangendo: a facilitação na execução de instalações de águas pluviais, hidrossanitárias e de gases hospitalares (VERNIZ; FABRÍCIO, 2011); facilidade de manutenção; uso de paredes divisórias removíveis; e, vantagens econômicas ocasionadas pela modulação para confecção de peças (DALLA CORTE; BERTUZZI; CARDOZO, 2018).

Finalizando as questões econômicas, três documentos debatem sobre o planejamento preditivo e seu potencial em reduzir custos, em contraste com os custos para remediar situações e consequências futuras (BRASIL, 1995c, 1995e, 2003). Em Brasil (1995c), ainda é discutido sobre como os investimentos contra incêndio, que são o foco da publicação, podem ser considerados itens sem retorno financeiro.

Enfim, sobre a sustentabilidade social, apesar desta tratar propriamente sobre a vida humana, estando rigorosamente ligada a função dos EAS, não foram encontrados atributos dessa esfera em duas publicações (BRASIL, 1995e, 1995f).

Considerando as publicações que buscaram ser sustentáveis quanto a esfera social, no geral, essa característica foi identificada pelo foco em preservar a vida e a promoção da saúde (BRASIL, 1995a, 1995c, 1995d, 2003, 2004). Esse é o caso de Brasil (1995d) que visou a otimização dos elementos técnicos por meio de projetos adequados de diversos tipos de instalações, de modo que o estabelecimento hospitalar ofereça as melhores condições para o pronto atendimento médico-hospitalar. Essa busca pode ser relacionada a meta 3.8 da ODS 3 da ONU, que objetiva o acesso a serviços de saúde essenciais de qualidade (ONU, 2015).

Brasil (1995g) e Brasil (1995h) englobaram o conforto do usuário, visando uma maior qualidade ambiental ao ocupante estando de acordo com a definição da sustentabilidade social. Foram incluídos critérios como o conforto higrotérmico, acústico e de iluminação ponderando sobre como o EAS deve promover a adequada satisfação às exigências do usuário. A questão do conforto ambiental em EAS também fez parte de outras publicações atuais como Castro, Mateus e Bragança (2017), Tang et al. (2019a) e Xuan (2018) que comparou o 
desempenho de EAS certificadas e não certificadas pela certificação ambiental Leadership in Energy and Environmental Design (LEED), e mostrou que as estratégias LEED implementadas podem ter um efeito positivo nas percepções da equipe sobre o ambiente interno, contribuindo para uma melhora na qualidade do serviço prestado.

Em Brasil (1995b) a preocupação com o suporte cognitivo da edificação hospitalar pode estar relacionada com o conforto visual do ocupante, item que faz parte do modelo de avaliação de EAS proposto por Castro, Mateus e Bragança (2017). Brasil (1995b) ainda trata da conexão do EAS com o lote englobando itens como a distância ao transporte coletivo são incluídos no documento. Uma publicação recente fez uma revisão das ferramentas de avaliação de desempenho pós-ocupação para avaliação hospitalar e identificou que a acessibilidade aos meios de transporte coletivo fez parte de 11\% dessas (BRAMBILLA; CAPOLONGO, 2019).

Assim, a busca pela sustentabilidade social pode estar alinhada com a busca pela humanização dos hospitais influenciando positivamente no tratamento dos pacientes, no bem-estar dos familiares e dos profissionais de saúde (LEITNER; PINA, 2020), sendo as janelas e jardins, por exemplo, itens que podem contribuir para a humanização do espaço (TISSOT; VERGARA; ELY, 2020), e os equipamentos, itens que podem influenciar negativamente nesse aspecto, como tratado em Brasil (2003).

Quanto a busca pela sustentabilidade real nos EAS, englobando assim suas três esferas, foram encontradas diversas características, ações e sugestões nas publicações ranqueadas pela ANVISA (ANVISA, 2020). Apesar disso, pode-se também refletir sobre a coesão entre as informações encontradas.

Entre as publicações, Brasil (1995c) mostrou-se o documento mais coeso na busca pela sustentabilidade global uma vez que discorra sobre as esferas econômicas, ambiental social sob o mesmo paradigma da prevenção de incêndio. Em outras palavras, no documento em questão, além de serem identificadas as três esferas da sustentabilidade as ações de cada esfera estão relacionadas de forma que o cumprimento de uma possa favorecer o cumprimento da outra. O mesmo acontece em Brasil (2003), o qual visa a segurança do trabalho de forma que suas ações possam trazer benefícios para as três esferas da sustentabilidade. No caso de Brasil (1995d) existe uma correlação entre as ações da esfera 
ambiental e social uma vez que a busca pela conservação de energia, tratada principalmente pelo viés econômico, pode trazer também benefícios ambientais.

Em outros casos não foram encontradas correlações entre as esferas da sustentabilidade, de forma que cada uma das esferas contribua para a busca pela sustentabilidade de forma isolada. Como exemplo, em Brasil (1995h) é discutido sobre o conforto do usuário demonstrando uma preocupação com a esfera social. Esse mesmo tópico poderia ser discutido nas esferas ambiental e econômica por meio da reflexão sobre como alcançar tal conforto indicando o uso de materiais, como mencionado em Brasil (1995d), e suas possíveis vantagens ambientais e econômicas. Outro exemplo, Brasil (1995h) engloba a questão das construções modulares e flexíveis, questão a qual poderia ter explorado as possíveis vantagens ambientais.

Existem ainda situações que ações que busquem uma esfera da sustentabilidade possam resultar em potenciais riscos, ou desvantagens, a outra esfera. Esse é o caso de Brasil (1995c) que discorre sobre uma situação que poderia representar, simultaneamente, uma vantagem econômica e um potencial risco a vida. Como descrito na publicação, o uso de materiais isolantes termoacústicos, que podem representar economia de energia, sendo uma vantagem econômica, e até mesmo ambiental, podem, em contrapartida, contribuir para o agravamento de um incêndio.

A situação descrita está prevista nas publicações uma vez que seja explicado que os critérios podem muitas vezes ser conflitantes entre si, cabendo ao responsável, seja ele arquiteto, engenheiro ou outro responsável técnico, avaliar aqueles mais adequados a sua situação.

A segunda parte dos resultados "What/ How" visou situar os documentos quanto a questão da pandemia do CoVID-19. Dentro desse contexto, o documento que mais se destacou foi Brasil (1995a) que aborda a arquitetura na prevenção de infecções hospitalares. Nele são descritos os tipos de isolamento sendo o chamado isolamento necessário aquele em casos de doenças infecciosa transmissível. Fazem parte do seu escopo: a preocupação com a lavagem das mãos; uso e transporte de roupas hospitalares; contaminação, limpeza e desinfecção dos dutos de ventilação; prevenção de incêndios e acidentes causados por mal uso do tubulão; entre outros. 
No documento em questão são descritas também ações que devem ser realizadas a fim de adicionar barreiras para a transmissão de doenças contagiosas, como a utilização de sacos solúveis, que se desintegram na lavagem das roupas minimizando o contato com os funcionários, sendo esse um risco biológico, como descrito em Brasil (2003), que também aborda o assunto. A questão também foi englobada em Brasil (1995c) por meio do uso de tubulão para a retirada de rejeitos, roupa contaminada etc.

Brasil (2003) também se destacou na questão do COVID-19 uma vez que apresente um capítulo dedicado a procedimentos de manutenção frente ao controle de infecção hospitalar. Nesse são ressaltados os cuidados de esterilização e desinfecção hospitalar, atividades as quais podem ter representativo impacto nos recursos da manutenção, sejam eles tempo ou dinheiro. Também é ressaltado o potencial dos ar-condicionados como agentes de contaminações, sendo que a importância da setorização do ar-condicionado também é abordada em Brasil (1995d).

Outro cuidado ressaltado em Brasil (2003), presente também em Brasil (1995c), é o emprego de revestimentos laváveis e que não possibilitem aderência da sujeira. Ainda sobre o assunto, Brasil (2004) discorre sobre a necessidade do local onde são guardados os resíduos, ou sala de resíduos, apresente paredes lisas e laváveis. Já Brasil (1995h) pondera sobre as condições do desempenho dos revestimentos e vedações em resistir agentes agressivos empregados em limpeza. Assim, diversas publicações tratam sobre a questão da limpeza dos EAS, fator esse que afeta diretamente o potencial de transmissão de doenças.

Em Brasil (1995b), o objetivo principal está relacionado com as questões visuais e arquitetônicas da edificação. No presente contexto, itens como "design diferenciado por ala/ função da edificação" podem, no dia a dia do funcionamento de um hospital gerenciando uma pandemia de uma doença contagiosa, evitar o contato desnecessário com pessoas ou objetos contaminados, minimizando transmissões. A importância da compartimentação da edificação também é abordada em Brasil (1995d) e em Brasil (1995c), que apesar de visar a contenção de incêndios, apresenta itens, como o afastamento mínimo entre torres de um hospital, que estão de acordo com a temática. Brasil (1995f) cita a necessidade de circulações duplas (limpa e suja) em algumas áreas de um hospital, mostrando mais uma vez a importância da compartimentação nos EAS. A publicação apresentada sugere que, quando a 
arquitetura permitir, a circulação suja seja feita como um corredor lateral aberto reduzindo em até 35\% dos custos de sua elaboração.

No caso de Brasil (1995e) não foram encontradas informações que pudessem ser relacionadas diretamente com o gerenciamento da pandemia do COVID-19. A publicação cita características como o uso de materiais lisos na construção de tubulões, como já citado por outras publicações, mas sem fazer associações com a facilidade de limpeza e possíveis transmissões contagiosas. Brasil (1995g) também não apresenta informações especificas para o problema. Sobre infecções hospitalares a publicação apenas refuta que o paisagismo possa ter algum efeito negativo sobre essa questão, citando como exemplo o Hospital do Aparelho Locomotor - Sarah Kubitscheck, localizado em Brasília, o qual apresenta as mais baixas taxas de infecção hospitalar apesar de densamente ajardinado. Com isso Brasil (1995g) salienta a importância da manutenção nos espaços internos.

Por fim, Brasil (2004), que engloba a importância do correto manejo dos resíduos, apresenta itens que podem auxiliar no gerenciamento de pandemias como o COVID-19, alguns sendo específicos para situações de risco por agentes biológicos, que é o caso do vírus. Entre esses itens estão:

- A higienização e limpeza em vigor no serviço, item que está relacionado também com a facilidade de limpeza ocasionada pela correta escolha dos materiais da construção (BRASIL, 1995c, 2003, 2004);

- A segurança ocupacional, que prevê ações como a imunização dos trabalhadores, em conformidade com o Programa Nacional de Imunização (PNI), item de suma importância no gerenciamento do COVID-19 quando a imunização estiver disponível;

- A capacitação dos trabalhadores quanto a importância da utilização correta de equipamentos de proteção individual (EPI) (uniforme, luvas, avental impermeável, máscara, botas e óculos de segurança específicos a cada atividade) bem como a necessidade de mantê-los em perfeita higiene e estado de conservação, sendo essa uma questão atual do COVID-19. 


\section{CONCLUSÃO}

O presente artigo visou, por meio da aplicação da ferramenta $5 \mathrm{~W} 1 \mathrm{H}$, identificar características intrínsecas dos EAS presentes nas publicações da ANVISA quanto a arquitetura e engenharia desse tipo de edificação. Seguindo o objetivo principal da pesquisa, foram detectadas dentro dos documentos ranqueados pela ANVISA, informações que pudessem ser relacionadas a busca pela sustentabilidade dos EAS englobando suas três esferas. Com isso, foi possível perceber que todos os documentos apresentaram alguma característica que pode ser relacionada com pelo menos uma das esferas da sustentabilidade, sendo que nove dos dez documentos preencheram os requisitos de duas das três esferas.

Entre as questões que se destacaram nos documentos, a que marcou a busca pela sustentabilidade ambiental foi o cuidado com contaminações ambientais e riscos ambientais (BRASIL, 1995b, 1995c, 1995e, 2003, 2004). Já o âmbito econômico esteve mais presente do que o anterior e permeou: questões energéticas, como o correto dimensionamento da rede e o consumo (BRASIL, 1995d, 1995e, 1995g); questões construtivas (BRASIL, 1995a, 1995f, 1995g, 1995h), englobando itens como a escolha do sistema construtivo, da volumetria da edificação e o uso de materiais isolantes, além de custos de manutenção (BRASIL, 1995h, 2003); e, o potencial do planejamento preditivo em reduzir custos (BRASIL, 1995c, 1995e, 2003). Por fim, a sustentabilidade social fez-se presente pelo foco em preservar a vida e a promoção da saúde (BRASIL, 1995a, 1995c, 1995d, 2003, 2004) e pela busca do conforto ambiental para o ocupante (BRASIL, 1995b, 1995g, 1995h).

Ademais, foi discutido ainda sobre como uma característica do EAS que envolve um dos pilares pode ou não auxiliar no cumprimento dos outros pilares. Assim, quanto a busca pela sustentabilidade real chegou-se à conclusão da importância de ações que possuam características da sustentabilidade ambiental, social e econômica concomitantemente de forma a evitar que ações que buscam as esferas da sustentabilidade isoladamente possam atritar entre si. Avaliando os itens que se destacaram em cada uma das esferas, e levando em consideração principalmente Brasil (1995h), o potencial das construções modulares e préfabricadas pode ser destacado de forma a alcançar: um maior conforto do usuário com melhores resultados térmicos e então energéticos; vantagens ambientais por meio da diminuição do consumo energético e uso de sistema construtivo com menor geração de resíduos do que a alvenaria convencional; vantagens econômicas com redução no tempo de 
obra, consumo energético, desperdícios, entre outros (BORJEGHALEH; SARDROUD, 2016; EL DEBS, 2000).

Posteriormente, foram ressaltados os fatores inerentes as construções hospitalares, como características arquitetônicas, que poderiam colaborar para o combate a pandemia do COVID-19, ou de outras doenças infecciosas. Dentre eles, destacam-se os apresentados a seguir:

1. Utilização de meios e tecnologias que evitem o contato direto entre funcionários, principalmente, e itens contaminados, constituindo barreiras para o contágio;

2. Uso de revestimentos, e materiais de construção em geral, de fácil limpeza e baixa aderência de sujeira auxiliando assim na limpeza, higienização e manutenção dos EAS;

3. Compartimentação do EAS, e do sistema de ar-condicionado, com a designação de alas especificas para doenças infeciosas de forma que o restante do EAS possa ser mantido sem riscos ou prejuízos ao seu funcionamento;

4. Capacitação dos funcionários a fim de abordar a importância da utilização correta de EPI e imunização da equipe, quando possível ou disponível.

Em suma, apesar da maioria das publicações ranqueadas no site da ANVISA para o uso orientativo da engenharia e arquitetura de EAS datarem cerca de 25 anos, foram encontradas informações que buscam a concepção e o funcionamento de um EAS tanto mais sustentável, quanto capaz de minimizar riscos em caso de doenças transmissíveis. Ainda assim, ressaltase a importância do desenvolvimento de norma especifica para construções de EAS, evitandose assim inconsistências e objetivando um melhor funcionamento do sistema de saúde, seja ele público ou privado.

Por fim, associando os resultados encontrados segundo os dois objetivos do presente estudo percebe-se que o cumprimento de itens do primeiro objetivo pode trazer também benefícios para o segundo. A instalação de um tubulão, por exemplo, citada por Brasil (1995a) por suas vantagens econômicas, auxilia na questão de evitar o contato entre funcionários e itens contaminados. Ademais, a busca pela sustentabilidade social marcada pela preocupação com a qualidade do serviço e manutenção da vida também é o foco do gerenciamento da 
pandemia do COVID-19. Assim, a busca por um EAS mais sustentável pode estar ligada a um EAS mais funcional e eficiente sendo mais apto a resolver situações não previstas.

\section{REFERÊNCIAS}

ASSOCIAÇÃO BRASILEIRA DE NORMAS TÉCNICAS (ABNT). NBR 15575-1: Edificações Habitacionais Desempenho Parte 1: Requisitos gerais. Rio de Janeiro, 2013.

BERNARDI, E.; CARLUCCI, S.; CORNARO, C.; BOHNE, R. A. An analysis of the most adopted rating systems for assessing the environmental impact of buildings. Sustainability, 9(7), 1226, 2017.

BONATTO, H. Reflexos da COVID-19 nas contratações de obras e serviços de engenharia: precisamos todos rejuvenescer. Olicitante, 2020. Disponível em: <https://www.olicitante.com.br/wpcontent/uploads/2020/04/REFLEXOS_DA_COVID-

19_NAS_CONTRATA\%C3\%87\%C3\%95ES_DE_OBRAS_E_SERVI\%C3\%870S_DE_ENGENHARIA2_Hamilton_Bonatto _O_Licitante.pdf>. Acesso em: 06 jul 2020.

BORJEGHALEH, R. M.; SARDROUD, J. M. Approaching Industrialization of Buildings and Integrated Construction Using Building Information Modeling. Procedia Engineering, v. 164, p. 534-541, 2016.

BRAMBILLA, A.; CAPOLONGO, S. Healthy and Sustainable Hospital Evaluation-A Review of POE Tools for Hospital Assessment in an Evidence-Based Design Framework. Buildings, v. 9, n. 4, p. 76, 2019.

BRASIL. Ministério da Saúde. Agência Nacional de Vigilância Sanitária (ANVISA). Arquitetura e Engenharia Manuais. Disponível em: <http://portal.anvisa.gov.br/arquitetura-e-engenharia-hospitalar>. Acesso em: 03 jul 2020.

BRASIL. Ministério da Saúde. Agência Nacional de Vigilância Sanitária (ANVISA). RESOLUÇÃo RDC № 306. Diário Oficial da União, Brasília, 2004.

Disponível em: <http://portal.anvisa.gov.br/documents/33880/2568070/res0306_07_12_2004.pdf/95eac678d441-4033-a5ab-f0276d56aaa6>. Acesso em: 14 jul 2020.

BRASIL. Ministério da Saúde. Agência Nacional de Vigilância Sanitária (ANVISA). RESOLUÇÃo RDC № 50/2002. Diário Oficial da União, Brasília, 2002. Disponível em: $<$ http://portal.anvisa.gov.br/documents/33880/2568070/res0050_21_02_2002.pdf/ca7535b3-818b-4e9d-907437c830fd9284>. Acesso em: 24 jul 2020.

BRASIL. Ministério da Saúde. Agência Nacional de Vigilância Sanitária (ANVISA). Segurança no ambiente hospitalar. $\quad 172 \quad \mathrm{p}$ 2003. 2 Disponível $<\underline{\text { http://portal.anvisa.gov.br/documents/33852/271121/seguranca_hosp.pdf/473c5e32-025a-4dc2-ab2e- }}$ fb5905d7233a>. Acesso em: 03 jul 2020.

BRASIL. Ministério da Saúde. Secretaria de Assistência à Saúde. Série Saúde \& Tecnologia - Textos de Apoio à Programação Física dos Estabelecimentos Assistenciais de Saúde - Arquitetura na Prevenção de Infecção Hospitalar. Brasília, $76 \quad p, \quad$ 1995a. Disponível em: <http://portal.anvisa.gov.br/documents/33852/271121/infeccao.pdf/c75403ca-3558-4a8c-a87a37c4ece21014>. Acesso em: 25 jun 2020.

BRASIL. Ministério da Saúde. Secretaria de Assistência à Saúde. Série Saúde \& Tecnologia - Textos de Apoio à Programação Física dos Estabelecimentos Assistenciais de Saúde - Condições Ambientais de Leitura Visual. 
Brasília, 90p, 1995b. Disponível em: http://portal.anvisa.gov.br/documents/33852/271121/ambientais.pdf/796fe21d-7517-4711-b46d6a531d336472>. Acesso em: 05 jul 2020.

BRASIL. Ministério da Saúde. Secretaria de Assistência à Saúde. Série Saúde \& Tecnologia - Textos de Apoio à Programação Física dos Estabelecimentos Assistenciais de Saúde - Condições de Segurança Contra Incêndio. Brasília, $107 \quad$ p, $1995 \mathrm{c} \quad$ Disponível em: http://portal.anvisa.gov.br/documents/33852/271121/incendio.pdf/97b95c4f-fc13-47a6-8ddd-033fb2b893be>. Acesso em: 05 jul 2020.

BRASIL. Ministério da Saúde. Secretaria de Assistência à Saúde. Série Saúde \& Tecnologia - Textos de Apoio à Programação Física dos Estabelecimentos Assistenciais de Saúde - Instalações Prediais Ordinárias e Especiais. Brasília, $61 \quad$ p, 1995d. Disponível em: http://portal.anvisa.gov.br/documents/33852/271121/prediais.pdf/1b3160c0-c788-439f-b508-00c4e67d9e3c>. Acesso em: 05 jul 2020.

BRASIL. Ministério da Saúde. Secretaria de Assistência à Saúde. Série Saúde \& Tecnologia - Textos de Apoio à Programação Física dos Estabelecimentos assistenciais de Saúde - Manutenção Incorporada à Arquitetura Hospitalar. Brasília, $74 \quad$ p, 1995e. Disponível em: http://portal.anvisa.gov.br/documents/33852/271121/manut.pdf/f27d475e-6db1-4445-a2a2-296c2ef9988a>. Acesso em: 05 jul 2020.

BRASIL. Ministério da Saúde. Secretaria de Assistência à Saúde Série Saúde \& Tecnologia - Textos de Apoio à Programação Física dos Estabelecimentos

Assistenciais de Saúde - O Custo das Decisões Arquitetônicas no Projeto de Hospitais. Brasília, 87 p,1995f. Disponível em: < http://portal.anvisa.gov.br/documents/33852/271121/custos.pdf/88b8bcb5-6dde-4dd7-840c3670324e34dd>. Acesso em: 05 jul 2020.

BRASIL. Ministério da Saúde. Secretaria de Assistência à Saúde Série Saúde \& Tecnologia - Textos de Apoio à Programação Física dos Estabelecimentos

Assistenciais de Saúde - Sistemas de Controle das Condições Ambientais de Conforto. Brasília, 92 p,1995g. Disponível em: <http://portal.anvisa.gov.br/documents/33852/271121/conforto.pdf/27660907-df0d-41aabaa1-e09f86116fd8>. Acesso em: 14 jul 2020.

BRASIL. Ministério da Saúde. Secretaria de Assistência à Saúde Série Saúde \& Tecnologia - Textos de Apoio à Programação Física dos Estabelecimentos

Assistenciais de Saúde - Sistemas Construtivos na Programação Arquitetônica de Edifícios de Saúde. Brasília, 53 p,1995h. Disponível em: <http://portal.anvisa.gov.br/documents/33852/271121/sistemas.pdf/4aec5696-277c-4db1-9027d85d8a0a8ac3>. Acesso em: 14 jul 2020.

CASTRO, M. de F.; MATEUS, R.; BRAGANÇA, L. Healthcare building sustainability assessment tool-sustainable effective design criteria in the Portuguese context. Environmental Impact Assessment Review, v. 67, p. 49-60, 2017.

CAVALCANTI, P. B.; ELY, V. H. M. B.; JEREMIAS, D. M.; SCHNEIDER, J. P.; SILVA, M. V.; BORTOLUZZI, T. V. C. Avaliação pós-ocupação de unidades de emergência hospitalares de Florianópolis: problemas recorrentes e possíveis soluções. Ambiente Construído, v. 19, n. 2, p. 171-186, 2019.

DALLA CORTE, C.; BERTUZZI, F. B.; CARDOSO, G. T. ESTRATÉGIAS BIOCLIMÁTICAS EM AMBIENTES HOSPITALARES: Um comparativo entre duas obras do arquiteto João Filgueiras Lima (Lelé). In: Seminário Internacional de Construções Sustentáveis (SICS), 7., 2018, Passo Fundo. Anais... Passo Fundo: IMED, 2018.

EL DEBS, M. K. Concreto pré-moldado: fundamentos e aplicações. São Carlos, SP: ESC/USP, 2000.

ELKINGTON, J. Towards the sustainable corporation: Win-win-win business strategies for sustainable development. California Management Review, v.36, n.2, p.90-100, 1994. 
FROEHLICH, C.; BITENCOURT, C. C. Sustentabilidade empresarial: um estudo de caso no Hospital Mãe de Deus. Sustentabilidade em debate, v. 6, n. 3, p. 100-115, set/dez 2015. doi:10.18472/SustDeb.v6n3.2015.15196

GIL, A. C. Como elaborar projetos de pesquisa. São Paulo: Atlas, 5 ed., 2010.

GUELLI, A. Tradução e adaptação do “AEDET - Achieving Excellence Design Evaluation Toolkit" para utilização no Brasil - Sistema de Avaliação Integral de Edifícios de Saúde. 2006. 42p. Dissertação (Mestrado Profissional em Economia da Saúde) - Universidade Federal de São Paulo, São Paulo, 2006. Disponível em: <http://www.repositorio.unifesp.br/handle/11600/21370>. Acesso em: 20 jul 2020.

INTERNATIONAL COUNCIL FOR RESEARCH AND INNOVATION IN BUILDING AND CONSTRUCTION (CIB). Agenda 21 on Sustainble Construction. CIB Report Publication 237. Rotterdam: CIB, July 1999.

LEE, J.; KIM, J.; AHN, J.; WOO, W. Context-aware risk management for architectural heritage using historic building information modeling and virtual reality. Journal of Cultural Heritage, v. 38, p. 242-252, 2019.

LEITNER, A. D.; PINA, S. M. Arquitetura sob a ótica da humanização em ambientes de quimioterapia pediátrica. Ambiente Construído, v. 20, n. 3, p. 179-198, 2020.

LEITNER, D. S.; SOTSEK, N. C.; SANTOS, A. P. L. Post occupancy Evaluation in Buildings: Systematic Literature Review. Journal of Performance of Constructed Facilities, v. 34, n. 1, p. 03119002, 2020. DOI.10.1061/(ASCE)CF.1943-5509.0001389

LOBO, A.V.R. Ferramenta de avaliação de sustentabilidade ambiental em edificações hospitalares na região metropolitana de Curitiba. 2010. 269 f. Dissertação (Mestrado em Construção Civil) - Universidade Federal do Paraná, Curitiba, 2010.

ORGANIZAÇÃO DAS NAÇÕES UNIDAS (ONU). Carta da Terra. 2002. Disponível em: <https://www.mma.gov.br/estruturas/agenda21/_arquivos/folder_carta_da_terra.pdf >. Acesso em: 08 jul 2020.

ORGANIZAÇÃO DAS NAÇÕES UNIDAS (ONU). Agenda 2030. 2015. Disponível em: <https://nacoesunidas.org/pos2015/agenda2030/>. Acesso em: 08 jul 2020.

PRINCIPI, P.; ROBERTO, F.; CARBONARI, A.; LEMMA, M. Evaluation of energy conservation opportunities through Energy Performance Contracting: A case study in Italy. Energy and Buildings, v. 128, p. 886-899, 2016.

REIJULA, J.; REIJULA, E; REIJULA, K. Insight into healthcare design: lessons learned in two university hospitals. Journal of Facilities Management, 2016.

RYAN-FOGARTY, Y.; O'REGAN, B.; MOLES, R. Greening healthcare: systematic implementation of environmental programs in a university teaching hospital. Journal of Cleaner Production, v. 126, p. 248-259, 2016.

SACHS, I. Estratégias de transição para o século XXI: desenvolvimento e meio ambiente. Studio Nobel, Fundação do Desenvolvimento Administrativo, 1993.

VERNIZ, D;; FABRÍCIO, M. M. INDUSTRIALIZAÇÃO DAS CONSTRUÇÕES: ANÁLISE DE OBRA HOSPITALAR. In: SIMPÓSIO BRASILEIRO DA GESTÃO E ECONOMIA DA CONSTRUÇÃO (SIBRAGEC), 7., 2011, Belém, Para. Anais... Disponível em: <https://www.researchgate.net/profile/Debora_Verniz2/publication/339830355_Industrializacao_das_constr ucoes_Analise_de_obra_hospitalar_Building_industrialization_analysis_of_a_hospital_construction/links/5e6 7ce2f4585153fb3d5a54e/Industrializacao-das-construcoes-Analise-de-obra-hospitalar-Buildingindustrialization-analysis-of-a-hospital-construction.pdf>. Acesso em: 24 jul 2020.

VILHENA, J. M. Diretrizes para a sustentabilidade das edificações. Gestão \& tecnologia de projetos, v. 2, n. 1, p. 59-78, 2007. 
TANG, H.; DING, J.; LI, C.; LI, J. A field study on indoor environment quality of Chinese inpatient buildings in a hot and humid region. Building and Environment, v. 151, p. 156-167, 2019a.

TANG, N.; HU, H.; XU, F.; ZHU, F. Personalized safety instruction system for construction site based on internet technology. Safety Science, v. 116, p. 161-169, 2019 b.

TISSOT, J. T.; VERGARA, L. G. L.; ELY, V.H. M. B. Definição de atributos ambientais essenciais para a humanização em quartos de internação. Ambiente Construído, v. 20, n. 3, p. 541-551, 2020.

XUAN, X. Study of indoor environmental quality and occupant overall comfort and productivity in LEED-and non-LEED-certified healthcare settings. Indoor and Built Environment, v. 27, n. 4, p. 544-560, 2018.

\section{(cc) $\mathrm{EY}$}

Este trabalho está licenciado com uma Licença Creative Commons - Atribuição 4.0 Internacional. 